OPEN ACCESS

Edited by:

Emilio Badoer

Royal Melbourne Institute of Technology (RMIT) University, Australia

Reviewed by:

Maria Giovanna Scarale,

Casa Sollievo della Sofferenza (IRCCS), Italy

Júlio Cezar De Oliveira

Federal University of Mato

Grosso, Brazi

*Correspondence:

Jing Dong

dongjing6@hotmail.com

${ }^{\dagger}$ These authors have contributed equally to this work

Specialty section

This article was submitted to Integrative Physiology, a section of the journal

Frontiers in Physiology

Received: 25 July 2019 Accepted: 30 October 2019 Published: 19 November 2019

Citation:

Su K, Li Y, Zhang D, Yuan J, Zhang C, Liu Y, Song L, Lin Q, Li M and Dong J (2019) Relation of Circulating Resistin to Insulin Resistance in Type 2 Diabetes and Obesity: A Systematic Review and Meta-Analysis. Front. Physiol. 10:1399.

doi: 10.3389/fphys.2019.01399

\section{Relation of Circulating Resistin to Insulin Resistance in Type 2 Diabetes and Obesity: A Systematic Review and Meta-Analysis}

\author{
Kai-zhen Su ${ }^{1 \dagger}$, Yan-run Li ${ }^{1 \dagger}$, Di Zhang ${ }^{2}$, Jun-hua Yuan ${ }^{2}$, Cai-shun Zhang ${ }^{2}$, Yuan Liu' ${ }^{2}$, \\ Li-min Song ${ }^{2}$, Qian Lin ${ }^{2}$, Man-wen $L^{2}{ }^{2}$ and Jing Dong ${ }^{2,3 *}$ \\ ${ }^{1}$ Clinical Medicine Department, Medical College, Qingdao University, Qingdao, China, ${ }^{2}$ Special Medicine Department, \\ Medical College, Qingdao University, Qingdao, China, ${ }^{3}$ Physiology Department, Medical College, Qingdao University, \\ Qingdao, China
}

Background: Resistin, a cysteine-rich polypeptide encoded by the RETN gene, which plays an important role in many mechanisms in rodent studies, including lipid metabolism, inflammation and insulin resistance. Nevertheless, the relationship between resistin and insulin resistance in humans is under debate. The present study was designed to clarify the correlation between resistin and insulin resistance.

Methods: A systematic literature search was performed using PubMed, Embase and Cochrane Library until March 3, 2019 with the keywords "resistin" and "insulin resistance." Funnel plots and Egger's test were used to detect publication bias. A random-effects model was used to calculate the pooled effect size. Subgroup analysis and meta regression was performed to identify the sources of heterogeneity.

Results: Fifteen studies were included in our systematic review. Among them, 10 studies with Pearson coefficients were used for meta-analysis. We found resistin levels were weakly correlated with insulin resistance in those with T2DM and obesity $(r=0.21,95 \%$ Cl: $\left.0.06-0.35, l^{2}=59.7 \%, P=0.003\right)$. Nevertheless, subgroup analysis suggested that circulating resistin levels were significantly positively correlated with insulin resistance in individuals with hyperresistinemia $(\geq 14.8 \mathrm{ng} / \mathrm{ml})\left(r=0.52,95 \% \mathrm{Cl}: 0.35-0.68, l^{2}=\right.$ $0.0 \%, P=0.513)$. And there was no relationship between circulating resistin and insulin resistance in those with normal circulating resistin levels $(<14.8 \mathrm{ng} / \mathrm{ml})(r=0.08,95 \%$ $\left.\mathrm{Cl}:-0.01-0.18, l^{2}=0.0 \%, P=0.455\right)$. Publication bias was insignificant (Egger's test $P=0.592)$.

Conclusion: In T2DM and obese individuals, resistin levels were positively correlated with insulin resistance in those with hyperresistinemia, but not in those with normal circulating resistin levels.

Keywords: resistin, insulin resistance, relation, diabetes mellitus, obesity, hyperresistinemia 


\section{INTRODUCTION}

Type 2 diabetes, characterized by insulin resistance (IR), is a complex chronic disorder of which the prevalence increased markedly in recent years (DeFronzo et al., 2015). In 2015, 415 million people were estimated to have diabetes, more than $90 \%$ of whom had type 2 diabetes (GBD Disease Injury Incidence Prevalence Collaborators, 2016). Obesity currently affects more than 600 million people worldwide by recent estimates and it was also characterized by defects of insulin action (Lois and Kumar, 2009). As the common pathology in obesity and type 2 diabetes, IR is defined as a state in which cells fail to respond to insulin, resulting in the development of hyperglycemia (Sah et al., 2016). In clinical studies, concerned about the problem of compliance with hyperinsulinemic euglycemic clamps, HOMA (homeostasis model assessment) has been applied more widely to estimate insulin sensitivity (Stern et al., 2005).

Resistin, also known as FIZZ3 or adipose tissue-specific secretory factor (ADSF), is a cysteine-rich polypeptide encoded by the RETN gene (Wang et al., 2002). The hormone was first discovered in a screen for targets of thiazolidinediones (TZDs) in white adipose of mice; it is believed to play a significant role in the development of insulin resistance (Steppan et al., 2001). The structure and distribution of resistin in humans are quite different from that of rodents. Murine resistin is a 114-amino acid polypeptide primarily produced in adipose tissues (Muse et al., 2004). While in humans, it is proved to be a 108-amino acid polypeptide expressed in adipocytes, pancreatic cells, muscle, and mononuclear cells (Dietze et al., 2002; Minn et al., 2003; Patel et al., 2003). It is worth mentioning that peripheral blood mononuclear cells (PBMCs) are key producers of resistin in humans (Kusminski et al., 2005). Its role in proinflammatory processes has been demonstrated in several studies (Kaser et al., 2003; Bokarewa et al., 2005).

Consistent with their varying structures, several functions of resistin vary between human and rodents. Rodent studies showed a clear role of resistin in the development of insulin resistance. Transgenic overexpression or recombinant of resistin impaired insulin function, while administration of anti-resistin antibody and antisense oligonucleotide treatment improved insulin sensitivity in mice (Steppan et al., 2001). However, the role of resistin in insulin resistance remains controversial in humans. Several studies found positive correlations between resistin and insulin resistance in T2DM, obese and healthy individuals (AlHarithy and Al-Ghamdi, 2005; Hivert et al., 2008; Zaidi and Shirwany, 2015); this discovery appears to be supported by studies reporting significantly higher resistin levels in populations with T2DM (Heilbronn et al., 2004). Nevertheless, a substantial number of studies have failed to find correlations between resistin and insulin resistance (Gerber et al., 2005; Bu et al., 2012).

To date, no systematic reviews or meta-analysis has analyzed the association between resistin and insulin resistance in populations; therefore, we analyzed this association in the present study.

\section{METHODS}

\section{Search Strategy}

The following databases were searched by two of the authors independently until March 3, 2019: PubMed, Cochrane Library and Embase. Two groups of keywords and their $\mathrm{MeSH}$ terms searched in PubMed were used to locate the relevant studies: resistin (e.g., "adipocyte secreted factor," "adipocyte specific secreted factor," "adipose tissue specific secreted factor," "ADSF," "FIZZ3," "FIZZ3 protein," "found in inflammatory zone 3 protein," "protein FIZZ3," "Adipocyte Cysteine-Rich Secreted Protein FIZZ3," "Adipocyte Cysteine Rich Secreted Protein FIZZ3") and insulin resistance (e.g., "Resistance, Insulin," "Insulin Sensitivity," "Sensitivity, Insulin," "resistance, insulin”). In order to make sure that there were not any articles missed, we did not limit the starting years. In addition, manual searches of the references of relevant studies were also conducted.

\section{Inclusion and Exclusion Criteria}

The inclusion criteria were set as follows: (1) observational study or baseline data of an experimental study; (2) participants aged $\geq 18$ years with simple obesity, simple T2DM or both; (3) presence of correlation coefficients regarding resistin and insulin resistance measured using the homeostasis model assessmentinsulin resistance (HOMA-IR); (4) Pearson's or Spearman's tests used to perform the correlation analyses; (5) publication in English language. And the studies were excluded if they met the following criteria: (1) reviews, comments, protocols, meeting abstracts, case report, or letters; (2) the participants of the study had other disorders, such as metabolic syndrome, non-alcoholic fatty liver disease or polycystic ovary syndrome.

\section{Data Extraction and Quality Assessment}

For data extraction, two of the authors reviewed the titles and abstracts of articles and then screened the full texts independently. The data were collected after all disagreements were resolved. We extracted the basic information (first author, year of publication, sample size, country) and participant characteristics (age, gender, BMI, fasting blood glucose, resistin, HOMA-IR, insulin) from the articles. And the data were converted to uniform units as needed. The Pearson's correlation coefficient was also extracted to obtain the effect size. In addition, we collected the Spearman's correlation coefficients to perform the systematic review.

The quality assessment was conducted using NOS (NewcastleOttawa Scale), including eight items, respectively (four items for selection, one item for comparability, three items for outcome in the scale of cross sectional studies and four items for selection, one item for comparability, three items for exposure in the scale of case control studies); NOS was used for cross-sectional studies and case control studies (Stang, 2010). A study was awarded stars according to the requirement of various questions. To assess the quality of the studies, study scores (ranging from 0 to 9 for case control studies) were classified as "high quality" (scores $\geq 6$ ) or "low quality" (scores $<6$ ) for further analysis.

We also used the Cochrane Collaborations tool to assess the reporting quality in randomized clinical trials (Higgins 
et al., 2011). The list of items in the tool includes seven items: random sequence generation, allocation concealment, blinding of participants and personnel, blinding of outcome assessment, incomplete outcome data, selective reporting and other bias. Two authors performed the assessment independently according to the tool protocol.

\section{Statistical Analysis}

Pearson correlation coefficients were transformed into Fisher's $z$ values to calculate the variance and corresponding data, and then we conducted meta-analysis and calculated the 95\% confidence intervals (CI). Heterogeneity between studies was tested via $I^{2}$ tests and Cochran's $Q$ tests. Studies which met the criteria of $I^{2}>50 \%$ in $I^{2}$ tests and $p<0.10$ in Cochran's $Q$ tests simultaneously were defined as having significant heterogeneity. If $I^{2}<50 \%$ or $p>0.10$, studies were considered as having low heterogeneity. We adopted a random-effects model when there was significant heterogeneity and publication bias was evaluated using visual assessment of funnel plot asymmetry and
Egger's tests. In addition, subgroup analyses, meta-regression and sensitivity analyses were performed. We conducted all these statistical analyses using Stata software version 12.0.

\section{RESULTS}

\section{Search Results and Study Inclusion}

Initially, the literature search identified 3,460 potential studies. After duplication checking, review of titles, abstracts and full texts, 15 eligible studies were included in this systematic review. Among them, 10 studies reported the correlations between resistin levels and IR with Pearson coefficients were used for further meta-analysis. The flow diagram of the search procedure is presented in Figure 1.

\section{Study Characteristics}

In the studies of Zaidi et al., Jung et al., Stepien et al., and KaplonCieslicka et al., patients were divided into two groups according to their study designs, and the correlation coefficients were given,

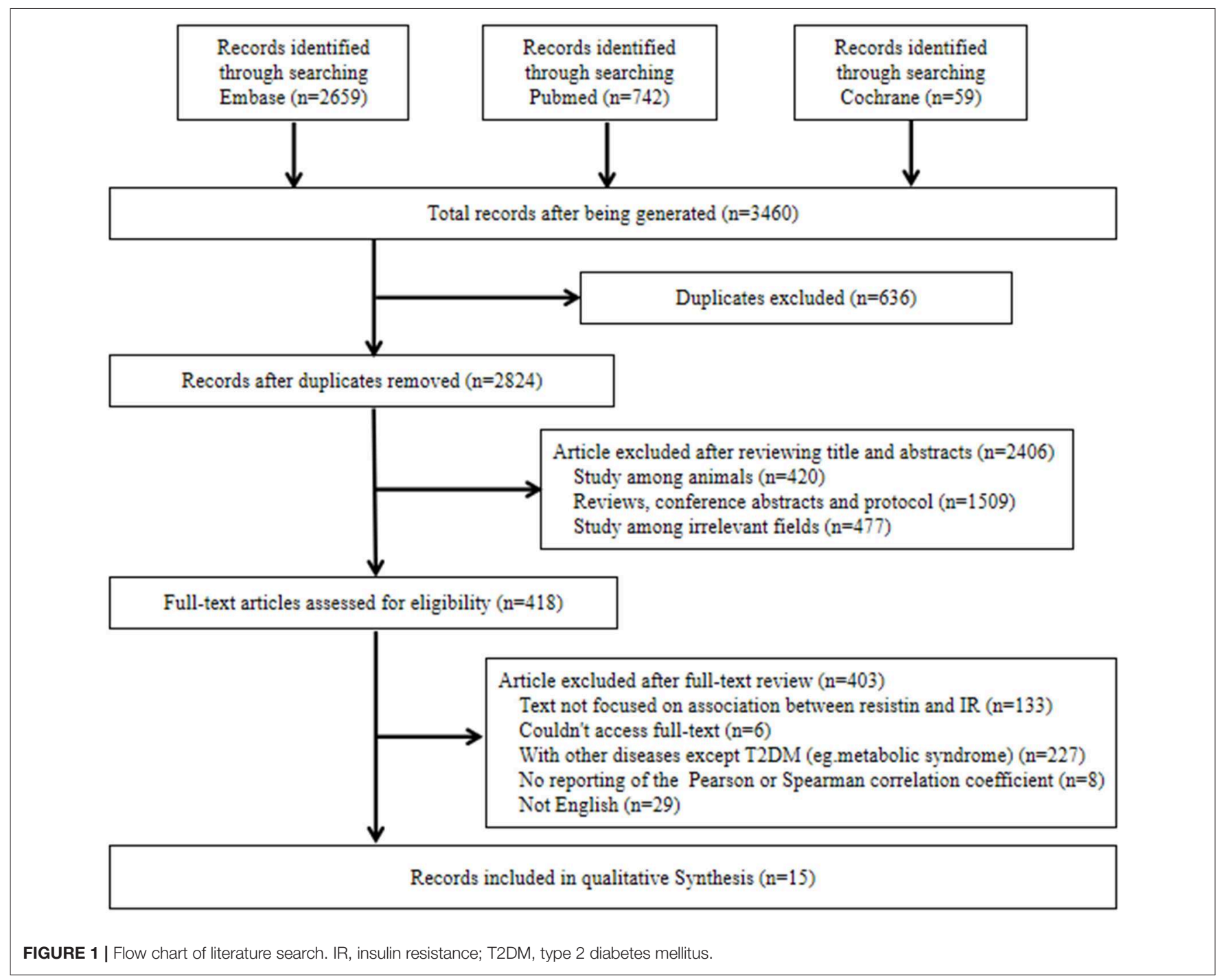


TABLE 1 | Characteristics of included studies.

\begin{tabular}{|c|c|c|c|c|c|c|c|c|c|c|c|c|c|c|}
\hline Author & Year & Country & $\begin{array}{l}\text { Study } \\
\text { design }\end{array}$ & $\begin{array}{l}\text { Study } \\
\text { population }\end{array}$ & $\begin{array}{l}\text { Number } \\
\text { (male/ } \\
\text { female) }\end{array}$ & Age & BMI & $\begin{array}{l}\text { Blood } \\
\text { glucose }\end{array}$ & Insulin & HOMA-IR & Resistin & $\begin{array}{l}\text { Correlation } \\
\text { coefficient } \\
\text { between } \\
\text { resistin } \\
\text { and IR }\end{array}$ & $\begin{array}{l}\text { Correlation } \\
\text { coefficient } \\
\text { type }\end{array}$ & $\begin{array}{l}\text { Blood } \\
\text { sample }\end{array}$ \\
\hline $\begin{array}{l}\text { Al-Harithy and } \\
\text { Al-Ghamdi } \\
\text { (2005) }\end{array}$ & 2005 & Saudi & $\begin{array}{l}\text { Cross } \\
\text { sectional }\end{array}$ & 44 & $0 / 44$ & $45 \pm 10$ & $25.5 \pm 4.9$ & $\begin{array}{l}8.74 \pm 4.04 \\
\mathrm{mmol} / \mathrm{l}\end{array}$ & $\begin{array}{l}12.96 \pm 5.15 \\
\mu \mathrm{IU} / \mathrm{mL}\end{array}$ & $5.38 \pm 6.63$ & $\begin{array}{l}19.42 \pm \\
3.60 \mathrm{ng} / \mathrm{ml}\end{array}$ & 0.49 & Pearson & Serum \\
\hline $\begin{array}{l}\text { Jung et al. } \\
\text { (2005) }\end{array}$ & 2005 & Korean & $R C T^{d}$ & 14 & $6 / 8$ & $60 \pm 8$ & $23.3 \pm 2.6$ & $\begin{array}{l}11.4 \pm 2.1 \\
\mathrm{mmol} / \mathrm{l}\end{array}$ & $\begin{array}{l}85 \pm 39 \\
\mathrm{pmol} / /\end{array}$ & $7.3 \pm 3.3$ & $\begin{array}{l}2.49 \pm \\
1.93 \mathrm{ng} / \mathrm{ml}\end{array}$ & 0.3 & Pearson & Plasma \\
\hline $\begin{array}{l}\text { Jung et al. } \\
\text { (2005) }\end{array}$ & 2005 & Korean & $R C T^{d}$ & 13 & $6 / 7$ & $54 \pm 14$ & $24.6 \pm 2.4$ & $\begin{array}{l}10.3 \pm 2.7 \\
\mathrm{mmol} / \mathrm{l}\end{array}$ & $\begin{array}{l}68 \pm 20 \\
\mathrm{pmol} / /\end{array}$ & $5.3 \pm 2.4$ & $\begin{array}{l}2.61 \pm \\
1.69 \mathrm{ng} / \mathrm{ml}\end{array}$ & 0.1 & Pearson & Plasma \\
\hline $\begin{array}{l}\text { Park et al. } \\
\text { (2006) }\end{array}$ & 2006 & Japan & $\begin{array}{l}\text { Cross } \\
\text { sectional }\end{array}$ & 104 & $53 / 51$ & $\begin{array}{l}53.8 \pm \\
9.2\end{array}$ & $23.87^{\mathrm{c}}$ & $\geq 7.0 \mathrm{mmol} / \mathrm{l}$ & - & $2.241^{c}$ & $6.527 \mathrm{ng} / \mathrm{ml}^{\mathrm{c}}$ & 0.157 & Pearson & Serum \\
\hline $\begin{array}{l}\text { Mojiminiyi and } \\
\text { Abdella } \\
\text { (2007) }\end{array}$ & 2007 & Kuwaiti & $\begin{array}{l}\text { Cross } \\
\text { sectional }\end{array}$ & 135 & $57 / 78$ & $58.92^{c}$ & $31.21^{\mathrm{C}}$ & $\begin{array}{l}9.928 \\
\mathrm{mmol} / /^{\mathrm{C}}\end{array}$ & $\begin{array}{l}21.239 \\
\mu \mathrm{IU} / \mathrm{mL}^{\mathrm{C}}\end{array}$ & $8.964^{c}$ & $23.61 \mathrm{ng} / \mathrm{mlc}$ & 0.3 & Spearman & Plasma \\
\hline $\begin{array}{l}\text { Tokuyama } \\
\text { et al. (2007) }\end{array}$ & 2007 & Japan & $\begin{array}{l}\text { Cross } \\
\text { sectional }\end{array}$ & 113 & $77 / 36$ & $\begin{array}{l}55.8 \pm \\
13.0\end{array}$ & $24.7 \pm 3.88$ & $\begin{array}{l}149.1 \pm 44.1 \\
\mathrm{mg} / \mathrm{dl}\end{array}$ & - & $3.82 \pm 5.16$ & $\begin{array}{l}10.35 \pm \\
6.30 \mathrm{ng} / \mathrm{ml}\end{array}$ & 0.302 & Spearman & Serum \\
\hline $\begin{array}{l}\text { Gharibeh } \\
\text { et al. (2010) }\end{array}$ & 2010 & Jordan & $\begin{array}{l}\text { Case } \\
\text { control }\end{array}$ & 140 & $63 / 77$ & $\begin{array}{l}54.36 \pm \\
0.88\end{array}$ & $\begin{array}{l}30.89 \pm \\
0.47\end{array}$ & $\begin{array}{l}10.10 \pm 0.32 \\
\mathrm{mmol} / \mathrm{l}\end{array}$ & $\begin{array}{l}11.94 \pm 0.60 \\
\mu \mathrm{IU} / \mathrm{mL}\end{array}$ & $5.46 \pm 0.37$ & $\begin{array}{l}7.82 \pm \\
0.29 \mathrm{ng} / \mathrm{ml}\end{array}$ & 0.08505 & Pearson & Plasma \\
\hline $\begin{array}{l}\text { Owecki et al. } \\
\text { (2011) }\end{array}$ & 2011 & Poland & $\begin{array}{l}\text { Cross } \\
\text { sectional }\end{array}$ & 136 & $61 / 75$ & $\begin{array}{l}48.8 \pm \\
13.4\end{array}$ & $\begin{array}{l}37.69 \pm \\
7.22\end{array}$ & $\begin{array}{l}5.49 \pm 0.63 \\
\mathrm{mmol} / \mathrm{l}\end{array}$ & $\begin{array}{l}40.62 \pm \\
54.04 \mu \mathrm{IU} / \mathrm{mL}\end{array}$ & - & $\begin{array}{l}24.89 \pm \\
9.73 \mathrm{ng} / \mathrm{ml}\end{array}$ & -0.1608 & Spearman & Serum \\
\hline $\begin{array}{l}\text { Stepien et al. } \\
\text { (2011) }\end{array}$ & 2011 & Poland & $\begin{array}{l}\text { Cross } \\
\text { sectional }\end{array}$ & 19 & $4 / 15$ & $\begin{array}{l}53.0 \pm \\
13.19\end{array}$ & $\begin{array}{l}33.19 \pm \\
3.13\end{array}$ & $\begin{array}{l}5.46 \pm 0.50 \\
\mathrm{mmol} / \mathrm{l}\end{array}$ & $\begin{array}{l}6.10 \pm 1.13 \\
\mu \mathrm{IU} / \mathrm{mL}\end{array}$ & $1.47 \pm 0.27$ & $\begin{array}{l}0.93 \pm \\
0.42 \mathrm{ng} / \mathrm{ml}\end{array}$ & -0.2000 & Spearman & Serum \\
\hline $\begin{array}{l}\text { Stepien et al. } \\
\text { (2011) }\end{array}$ & 2011 & Poland & $\begin{array}{l}\text { Cross } \\
\text { sectional }\end{array}$ & 18 & $7 / 11$ & $\begin{array}{l}49.56 \pm \\
14.16\end{array}$ & $\begin{array}{l}34.77 \pm \\
3.52\end{array}$ & $\begin{array}{l}5.72 \pm 0.52 \\
\mathrm{mmol} / \mathrm{l}\end{array}$ & $\begin{array}{l}14.29 \pm 4.68 \\
\mu \mathrm{IU} / \mathrm{mL}\end{array}$ & $3.64 \pm 1.28$ & $\begin{array}{l}1.04 \pm \\
0.44 \mathrm{ng} / \mathrm{ml}\end{array}$ & 0.1662 & Spearman & Serum \\
\hline $\begin{array}{l}\text { Bu et al. } \\
\text { (2012) }\end{array}$ & 2012 & China & $\begin{array}{l}\text { Cross } \\
\text { sectional }\end{array}$ & 22 & $10 / 12$ & $49.64^{c}$ & $22.75^{\mathrm{C}}$ & $\begin{array}{l}7.736 \\
\mathrm{mmol} / /^{\mathrm{C}}\end{array}$ & $\begin{array}{l}7.345 \\
\mu \mathrm{IU} / \mathrm{mL}^{\mathrm{c}}\end{array}$ & $2.013^{\mathrm{C}}$ & $1.717 \mathrm{ng} / \mathrm{ml}^{\mathrm{C}}$ & -0.178 & Pearson & Plasma \\
\hline $\begin{array}{l}\text { Yin et al. } \\
\text { (2012) }\end{array}$ & 2012 & China & $\begin{array}{l}\text { Case } \\
\text { control }\end{array}$ & 38 & $18 / 20$ & $45 \pm 10$ & $25.5 \pm 4.9$ & $\begin{array}{l}7.4 \pm 1.7 \\
\mathrm{mmol} / \mathrm{l}\end{array}$ & $\begin{array}{l}9.2 \pm 6.2 \\
\mathrm{mlU} / \mathrm{l}\end{array}$ & $5.5^{\mathrm{c}}$ & $\begin{array}{l}12.3 \pm \\
2.7 \mathrm{ng} / \mathrm{ml}\end{array}$ & 0.09 & Pearson & Serum \\
\hline $\begin{array}{l}\text { Mabrouk } \\
\text { et al. (2013) }\end{array}$ & 2013 & Egypt & $\begin{array}{l}\text { Case } \\
\text { control }\end{array}$ & 24 & $8 / 16$ & $32.4 \pm 9$ & $53.7 \pm 5.43$ & $\begin{array}{l}161.8 \pm 23.9 \\
\mathrm{mg} / \mathrm{dl}\end{array}$ & $\begin{array}{l}17.7 \pm 1.8 \\
\mu \mathrm{IU} / \mathrm{mL}\end{array}$ & $5.4 \pm 0.65$ & $\begin{array}{l}51 \pm \\
8.2 \mathrm{ng} / \mathrm{ml}\end{array}$ & 0.56 & Pearson & Serum \\
\hline $\begin{array}{l}\text { Stepien et al. } \\
\text { (2014) }\end{array}$ & 2014 & Poland & $\begin{array}{l}\text { Cross } \\
\text { sectional }\end{array}$ & 16 & $4 / 12$ & $\begin{array}{l}59.3 \pm \\
13.4^{\mathrm{a}}\end{array}$ & $34.5 \pm 3.0^{\mathrm{a}}$ & $\begin{array}{l}5.1 \pm 0.4 \\
\mathrm{mmol} / \mathrm{I}^{\mathrm{a}}\end{array}$ & $\begin{array}{l}7.5 \pm 2.3 \\
\mu \mathrm{IU} / \mathrm{mL}^{\mathrm{a}}\end{array}$ & $1.7 \pm 0.5^{\mathrm{a}}$ & $\begin{array}{l}1.0 \pm 0.4 \\
\mathrm{ng} / \mathrm{ml}^{\mathrm{a}}\end{array}$ & 0.013 & Pearson & Serum \\
\hline $\begin{array}{l}\text { Stepien et al. } \\
\text { (2014) }\end{array}$ & 2014 & Poland & $\begin{array}{l}\text { Cross } \\
\text { sectional }\end{array}$ & 48 & $20 / 28$ & $\begin{array}{l}54.8 \pm \\
11.2\end{array}$ & $36.8 \pm 4.9$ & $\begin{array}{l}5.9 \pm 0.4 \\
\mathrm{mmol} / \mathrm{l}\end{array}$ & $\begin{array}{l}17.2 \pm 8.3 \\
\mu \mathrm{IU} / \mathrm{mL}\end{array}$ & $4.5 \pm 2.3$ & $\begin{array}{l}1.0 \pm \\
0.3 \mathrm{ng} / \mathrm{ml}\end{array}$ & -0.223 & Pearson & Serum \\
\hline $\begin{array}{l}\text { Zaidi and } \\
\text { Shirwany } \\
\text { (2015) }\end{array}$ & 2015 & Pakistan & $\begin{array}{l}\text { Cross } \\
\text { sectional }\end{array}$ & 40 & $12 / 28$ & $44 \pm 7$ & $35 \pm 5$ & $\begin{array}{l}164 \pm 46 \\
\mathrm{mg} / \mathrm{dl}\end{array}$ & $\begin{array}{l}37 \pm 7 \\
\mu \mathrm{IU} / \mathrm{mL}\end{array}$ & $19 \pm 8$ & $38 \pm 8 \mathrm{ng} / \mathrm{ml}$ & 0.561 & Pearson & Serum \\
\hline $\begin{array}{l}\text { Zaidi and } \\
\text { Shirwany } \\
\text { (2015) }\end{array}$ & 2015 & Pakistan & $\begin{array}{l}\text { Cross } \\
\text { sectional }\end{array}$ & 40 & $12 / 28$ & $40 \pm 6$ & $33 \pm 3$ & $83 \pm 8 \mathrm{mg} / \mathrm{dl}$ & $\begin{array}{l}26 \pm 6 \\
\mu \mathrm{IU} / \mathrm{mL}\end{array}$ & $5 \pm 1$ & $25 \pm 5 \mathrm{ng} / \mathrm{ml}$ & 0.307 & Pearson & Serum \\
\hline
\end{tabular}




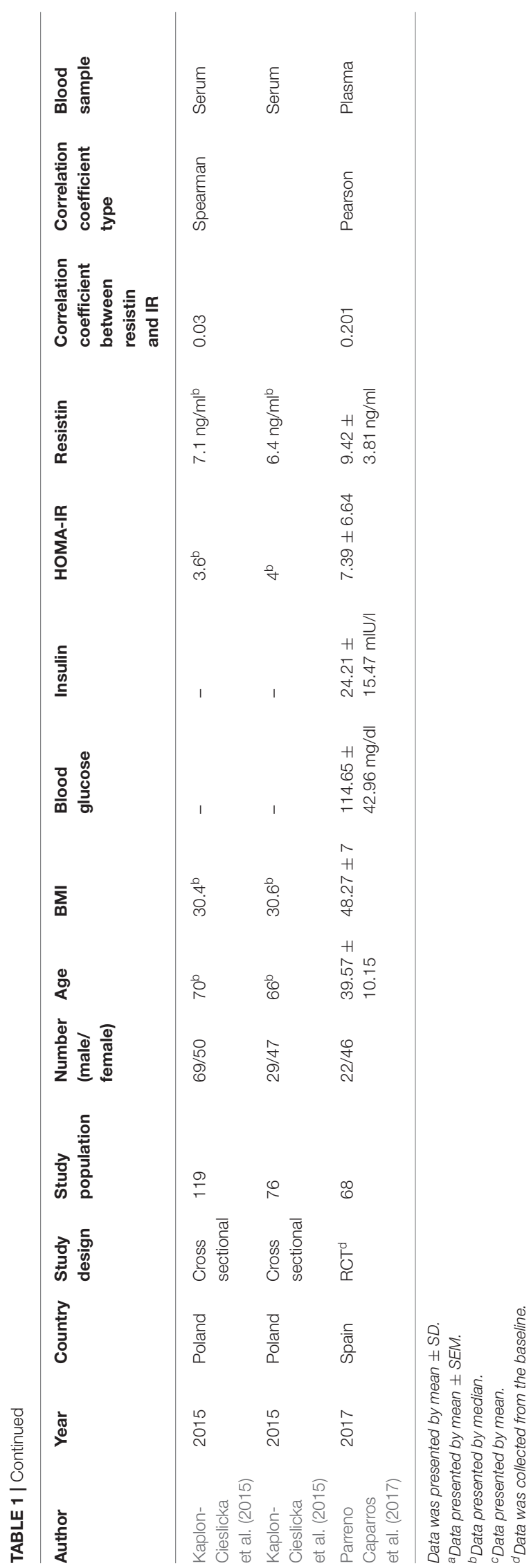

respectively (Jung et al., 2005; Stepien et al., 2014; KaplonCieslicka et al., 2015; Zaidi and Shirwany, 2015). Therefore, we included 15 studies with 20 groups, a total of 1,227 patients. Seven of the groups were simple obesity (Owecki et al., 2011; Stepien et al., 2011, 2014; Zaidi and Shirwany, 2015; Parreno Caparros et al., 2017), ten of them were simple T2DM (Al-Harithy and Al-Ghamdi, 2005; Jung et al., 2005; Park et al., 2006; Mojiminiyi and Abdella, 2007; Tokuyama et al., 2007; Gharibeh et al., 2010; Bu et al., 2012; Yin et al., 2012; Kaplon-Cieslicka et al., 2015), two of them were obesity with T2DM (Mabrouk et al., 2013; Zaidi and Shirwany, 2015), and one of them was T2DM with or without obesity. The publication years ranged from 2005 to 2017, and the sample sizes ranged from 13 to 140 . Fasting serum or plasma resistin levels were measured using enzyme linked immunosorbent assay (ELISA). More details of the included studies are displayed in Table $\mathbf{1}$.

\section{Overall Meta-Analysis}

Ten studies including 13 groups and 611 patients with Pearson coefficients were used for meta-analysis. As demonstrated in Figure 2, resistin levels were weakly correlated with IR ( $r=$ $0.21,95 \%$ CI: $0.06-0.35)$. The $r$ values exhibiting significant heterogeneity were evaluated using a random-effects model $\left(I^{2}=\right.$ $59.7 \%, P=0.003)$. No publication bias was found by inspection of funnel plots and using Egger's tests $(P=0.592)$ (Figure 3).

\section{Subgroup Meta-Analysis}

In order to explore the sources of heterogeneity, we performed subgroup analysis. A study from Japan $(n=3192)$ reported quartiles of blood resistin levels in the general population (Kawamura et al., 2010). We chose the $75 \%$ point to divide these studies into a hyperresistinemia group (blood resistin $\geq 14.8 \mathrm{ng} / \mathrm{ml}$ ) and a normal circulating resistin group (blood resistin $<14.8 \mathrm{ng} / \mathrm{ml}$ ). In the hyperresistinemia group, resistin levels significantly positively correlated with IR $(r=0.52$, 95\% CI: $\left.0.35-0.68, I^{2}=0.0 \%, P=0.513\right)$. However, the normal circulating resistin group did not show a significant correlation $\left(r=0.08,95 \%\right.$ CI: $-0.01-0.18, I^{2}=0.0 \%, P=$ $0.455)$ (Figure 4). After reviewing all the studies with Pearson or Spearman coefficients together, we found a similar result to this analysis. Therefore, we deduced that resistin levels were positively correlated with IR only in those with hyperresistinemia, but not in those with normal circulating resistin levels.

In the following work, we analyzed subgroups classified by Study design (RCT or cross sectional or case control) and blood sample (serum or plasma) (Table 2). However, none of these factors showed statistical significance or reduced heterogeneity in each subgroup.

\section{Meta-Regression}

Meta-regression analysis (Table 3) indicated that resistin is a significant influence factor of the correlation coefficient $(p=$ 0.001) (Figure 5). The results didn't support a relationship between the correlation coefficient and other influence factors including age, BMI, blood glucose and insulin ( $p>0.05$ for all). 


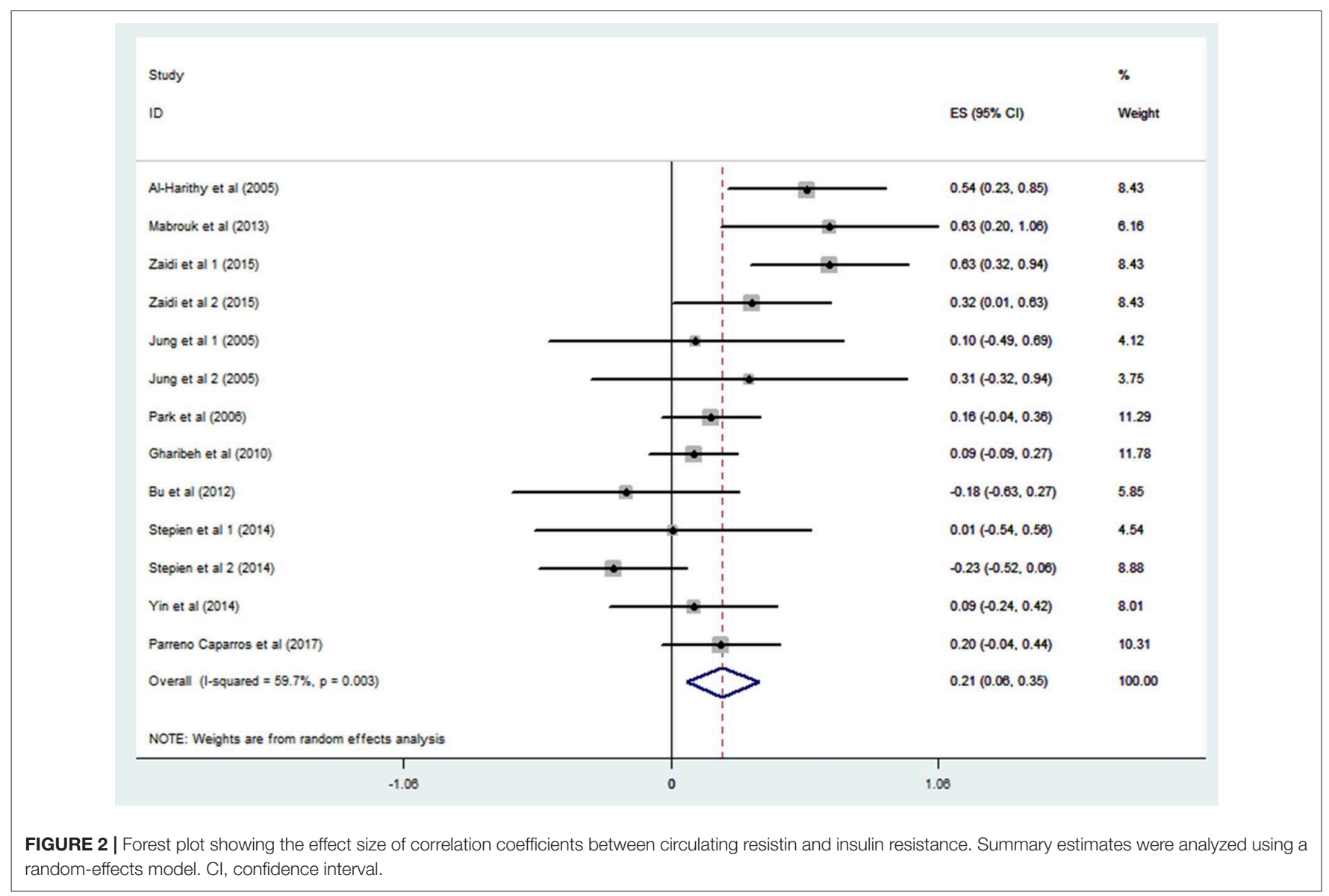

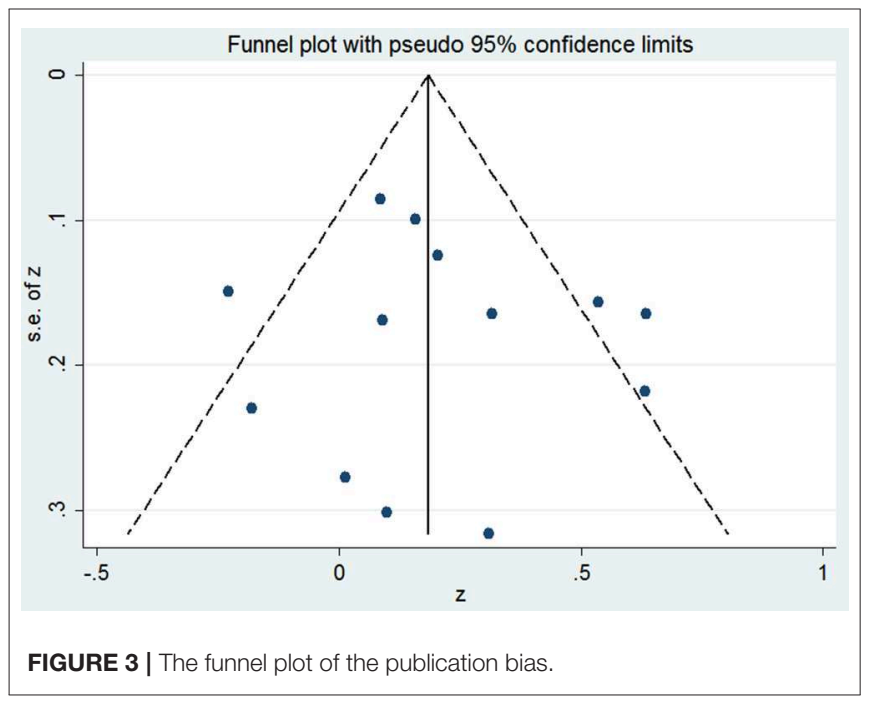

\section{DISCUSSION}

In the present meta-analysis, we found that resistin levels are correlated with insulin resistance in obese and T2DM patients, and this correlation was associated with resistin levels. To our knowledge, this is the first systematic review and meta-analysis using statistical methods to analyze the correlation between resistin levels and insulin resistance.

Many studies indicated that multiple adipokines, such as adiponectin and leptin were involved in the occurrence and development of insulin resistance, possibly associated with many complications (Hara et al., 2002; German et al., 2010). Resistin, an adipokine first discovered by Steppan in 2001, has been shown to induce insulin resistance in rodents. Increased resistin levels were also found in diet-induced or genetically obese mice (Steppan et al., 2001). In human studies, individuals with severe insulin resistance had higher resistin levels than individuals with normal insulin action (Zaidi and Shirwany, 2015). Therefore, we hypothesized that resistin might also play a role in insulin resistance, possibly providing new therapeutic targets for the treatment and prevention of diabetes.

Nevertheless, the relationship between resistin levels and insulin resistance has not been clarified in humans. In 2005, Al-Harithy et al. first reported a positive correlation between resistin levels and insulin resistance in diabetic women and obese or overweight non-diabetic women (Al-Harithy and Al-Ghamdi, 2005). A study in Pakistan also found a correlation in the diabetes groups and the obese non-diabetic groups (Zaidi and Shirwany, 2015). Moreover, Mabrouk et al. found a positive correlation only in obese diabetic Egyptian subjects, but not in obese non-diabetic groups (Mabrouk et al., 2013). There have also been several 


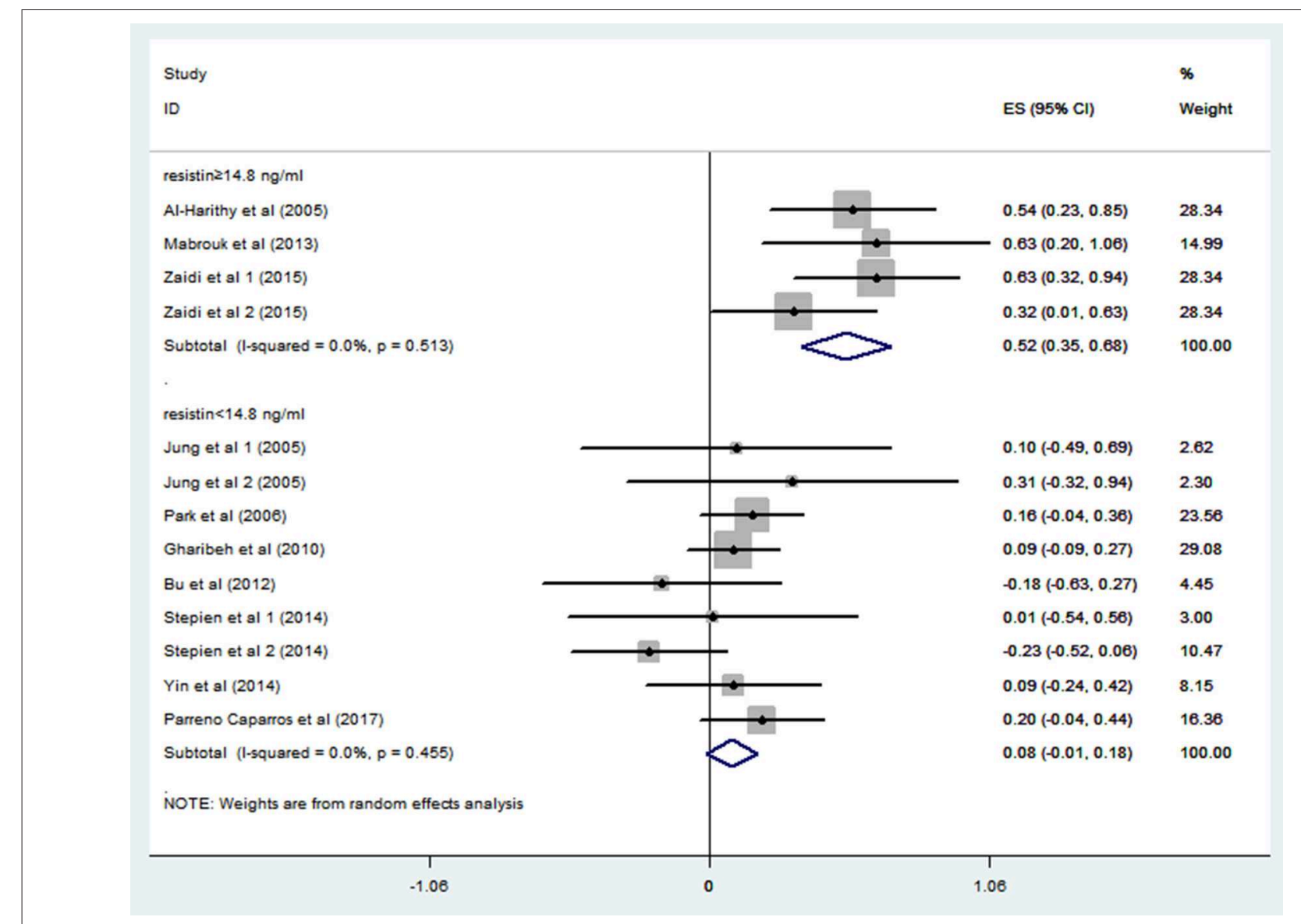

FIGURE 4 | Forest plot of correlation coefficients between circulating resistin and insulin resistance based on fasting circulating resistin levels. Cl, confidence interval.

studies failing to find a significant correlation between resistin levels and insulin resistance in diabetic groups. Bu et al. found resistin levels had no relationship to IR in both T2DM group and normoglycemic group (Bu et al., 2012). Park et al. also observed these results in a cross-sectional study (Park et al., 2006). More and more studies concentrated on this study orientation obtained conflicting results (Mojiminiyi and Abdella, 2007; Stepien et al., 2011; Yin et al., 2012). Therefore, we attempted to reconcile these discrepancies and to draw a rational conclusion.

Among the articles searched from online databases, 15 met our selection criteria. Ten articles with Pearson's coefficients were used for meta-analysis. The overall analysis revealed a positive correlation between resistin levels and insulin resistance $(\mathrm{r}=$ $0.21,95 \%$ CI: $0.06-0.35)$; however, heterogeneity $\left(I^{2}=59.7 \%\right.$, $P=0.003)$ remained significant. Consequently, we performed subgroup analysis to identify the sources of heterogeneity. According to blood resistin levels, studies were divided according to hyperresistinemia groups $\left(r=0.52,95 \%\right.$ CI: $0.35-0.68, I^{2}=$ $0.0 \%, P=0.513)$ and normal circulating resistin groups $(r=0.08$, $95 \%$ CI: $\left.-0.01-0.18, I^{2}=0.0 \%, P=0.455\right)$. The heterogeneity was markedly low in both group, suggesting that resistin levels were a vital influence factor in this correlation. Applying this result to all included studies, only one study did not fit (Owecki et al., 2011). This may have resulted from the high standard difference of insulin in that study, a point also mentioned by the author. We further analyzed the subgroups classified by Study design and blood sample. None reached acceptable heterogeneity.

So far, the mechanisms of insulin-resistance related effects of resistin are not clear in humans. In vitro studies have demonstrated that recombinant human resistin could induce insulin resistance through $5^{\prime} \mathrm{AMP}$-activated protein kinase (AMPK)-dependent and AMPK-independent suppressor of cytokine signaling-3 (SOCS-3) signaling pathways in HepG2 cells (Luo et al., 2009), which is similar to the related rodent studies (Muse et al., 2004; Steppan et al., 2005). In addition, considering about the distribution of resistin in mononuclear cells in humans, inflammation might be the process which links resistin to insulin resistance. Previous studies have pointed out that resistin plays a role in pro-inflammatory processes. In vitro studies suggested that expression of resistin have been the result of the production of the pro-inflammatory cytokines, such as tumor necrosis factor- $\alpha$ (TNF- $\alpha$ ) and interleukin-6 (IL-6) (Kaser et al., 2003; Bokarewa et al., 2005), besides, resistin could also stimulates the expression of pro-inflammatory cytokines, including TNF- $\alpha$, IL-6 through the nuclear factor- $\mathrm{K}$ (NF- $\mathrm{\kappa} B, \mathrm{p} 50 / \mathrm{p} 65)$ signaling pathway (Silswal et al., 2005). Chronic inflammation is a major 
TABLE 2 | Subgroup analyses of correlations between insulin resistance and resistin.

\begin{tabular}{|c|c|c|c|c|c|}
\hline & Groups & Participants & $\begin{array}{l}\text { Random } \\
\text { effects } r \\
(95 \% \mathrm{Cl})\end{array}$ & $I^{2}(\%)$ & $\begin{array}{c}P \text { for } \\
\text { heterogeneity }\end{array}$ \\
\hline $\begin{array}{l}\text { Overall } \\
\text { Subgroup } \\
\text { analysis }\end{array}$ & 13 & 611 & $\begin{array}{c}0.21(0.06 \\
0.35)\end{array}$ & 59.7 & 0.003 \\
\hline \multicolumn{6}{|l|}{ Resistin } \\
\hline $\begin{array}{l}\text { Resistin } \geq \\
14.8 \mathrm{ng} / \mathrm{ml}\end{array}$ & 6 & 148 & $\begin{array}{c}0.52(0.35 \\
0.68)\end{array}$ & 0 & 0.513 \\
\hline $\begin{array}{l}\text { Resistin < } \\
14.8 \mathrm{ng} / \mathrm{ml}\end{array}$ & 7 & 463 & $\begin{array}{c}0.08(-0.01 \\
0.18)\end{array}$ & 0 & 0.455 \\
\hline \multicolumn{6}{|l|}{ Study design } \\
\hline Case control & 3 & 202 & $\begin{array}{c}0.22(-0.07 \\
0.51)\end{array}$ & 64.2 & 0.061 \\
\hline $\begin{array}{l}\text { Cross } \\
\text { sectional }\end{array}$ & 7 & 314 & $\begin{array}{c}0.20(-0.05 \\
0.44)\end{array}$ & 74.7 & 0.001 \\
\hline $\mathrm{RCT}$ & 3 & 95 & $\begin{array}{c}0.20(-0.01 \\
0.41)\end{array}$ & 0 & 0.892 \\
\hline \multicolumn{6}{|c|}{ Blood sample } \\
\hline Serum & 8 & 354 & $\begin{array}{c}0.27(0.05 \\
0.48)\end{array}$ & 71.7 & 0.001 \\
\hline Plasma & 5 & 257 & $\begin{array}{c}0.11(-0.02 \\
0.23)\end{array}$ & 0 & 0.618 \\
\hline
\end{tabular}

$R C T$, randomized control trial; $\mathrm{Cl}$, confidence interval.

TABLE 3 | Meta-regression of correlations between insulin resistance and resistin.

\begin{tabular}{lcccc}
\hline & Coefficient $\boldsymbol{\beta}$ & SE & $\mathbf{9 5 \%} \mathbf{C l}$ & $\boldsymbol{P}$-value \\
\hline Insulin & 0.012 & 0.007 & $(-0.004,0.028)$ & 0.123 \\
Resistin & 0.016 & 0.003 & $(0.008,0.024)$ & 0.001 \\
Glucose & 0.020 & 0.026 & $(-0.037,0.076)$ & 0.465 \\
Age & -0.016 & 0.009 & $(-0.037,0.004)$ & 0.099 \\
BMl & 0.010 & 0.009 & $(-0.008,0.029)$ & 0.246
\end{tabular}

BMI, body mass index.

and well-known cause of obesity-induced insulin resistance (Gregor and Hotamisligil, 2011), and several pro-inflammatory cytokines play an important role in the process. For example, the TNF- $\alpha$ signaling pathway activates intracellular kinases that inhibit insulin receptor signaling via serine phosphorylation of insulin receptor substrate 1 (IRS-1) (Hotamisligil et al., 1993), IL6 and adiponectin also regulate insulin sensitivity (Maeda et al., 2002; Weigert et al., 2006). Resistin might exert similar functions in light of its distribution and inflammation-related functions.

In subgroup analysis, resistin levels positively correlated with insulin resistance only in people with higher resistin levels, but not in those with lower resistin levels, which was consistent with results reported by Al-Harithy and Al-Ghamdi (2005) and Mabrouk et al. (2013). The meta regression also indicated resistin levels is the source of heterogeneity. This might be explained by the hypothesis that resistin might be the principal factor which induced insulin resistance at high levels. However, the

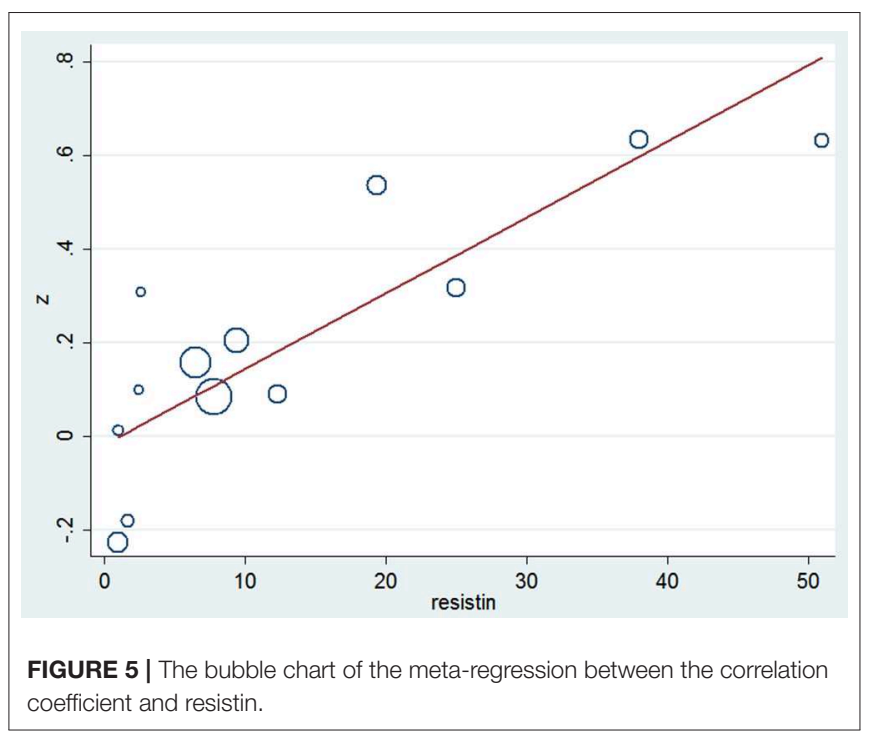

overall effect of resistin was not significant in people with low resistin levels, several other factors may dominate insulin resistance like free fatty acids, hyperosmotic stress, and TNF$\alpha$ (Roden et al., 1996, 1999; Gual et al., 2003; Plomgaard et al., 2005). In the study of Park et al., people with insulin resistance did not have a correlation between resistin and insulin resistance in the case of low resistin levels, but their insulin resistance significantly correlated with TNF- $\alpha$, which meet the hypothesis above (Park et al., 2006). There are also several other hypotheses regarding the controversial correlation between resistin and insulin resistance. Several single nucleotide polymorphisms (SNPs) of the resistin RETN gene have been reported to be associated with resistin concentration, while this association remains controversial in various ethnicities, possibly explaining the conflicting results of the correlation (Hivert et al., 2009). Zaidi et al. suggested that resistin causes insulin resistance when insulin reaches a certain critical level (Zaidi and Shirwany, 2015), but we did not draw this conclusion in our analysis, which may due to the limited number of relevant studies. More studies are needed to verify these hypotheses in the future.

A number of treatments targeting adipokines have recently emerged. For example, monoclonal antibody infliximab, which neutralizes TNF- $\alpha$, is a new approach aimed at inflammationassociated insulin resistance (Ursini et al., 2010). That study indicated that resistin might be a therapy targeting insulin resistance in the patients with hyperresistinemia. A new therapy to reduce resistin levels may alleviate insulin resistance in the future.

Limitations in our meta-analysis should be considered. There were some studies with small sample sizes included in the present analysis, possibly explaining the heterogeneity. Few studies concentrating on the correlation between resistin and insulin resistance without reporting the correlation coefficient may have introduced bias. Some non-English articles were also 
excluded in our study. Therefore, larger clinical trials are needed to further validate our results.

\section{CONCLUSIONS}

In T2DM and obese individuals, resistin levels are positively correlated with insulin resistance in people with hyperresistinemia, but not in those with normal circulating resistin levels. This study may help to suggest new targets for the prevention and treatment of insulin resistance.

\section{AUTHOR CONTRIBUTIONS}

KS and YL designed the program, searched and reviewed the studies, were in charge of the manuscript. YL and LS assessed the

\section{REFERENCES}

Al-Harithy, R. N., and Al-Ghamdi, S. (2005). Serum resistin, adiposity and insulin resistance in Saudi women with type 2 diabetes mellitus. Ann. Saudi Med. 25, 283-287. doi: 10.5144/0256-4947.2005.283

Bokarewa, M., Nagaev, I., Dahlberg, L., Smith, U., and Tarkowski, A. (2005). Resistin, an adipokine with potent proinflammatory properties. J. Immunol. 174, 5789-5795. doi: 10.4049/jimmunol.174.9.5789

Bu, J., Feng, Q., Ran, J., Li, Q., Mei, G., and Zhang, Y. (2012). Visceral fat mass is always, but adipokines (adiponectin and resistin) are diversely associated with insulin resistance in Chinese type 2 diabetic and normoglycemic subjects. Diabetes Res. Clin. Pract. 96, 163-169. doi: 10.1016/j.diabres.2011.12.014

DeFronzo, R. A., Ferrannini, E., Groop, L., Henry, R. R., Herman, W. H., Holst, J. J., et al. (2015). Type 2 diabetes mellitus. Nat. Rev. Dis. Primers. 1:15019. doi: $10.1038 /$ nrdp.2015.19

Dietze, D., Koenen, M., Rohrig, K., Horikoshi, H., Hauner, H., and Eckel, J. (2002). Impairment of insulin signaling in human skeletal muscle cells by co-culture with human adipocytes. Diabetes 51, 2369-2376. doi: $10.2337 /$ diabetes.51.8.2369

GBD Disease and Injury Incidence and Prevalence Collaborators (2016). Global, regional, and national incidence, prevalence, and years lived with disability for 310 diseases and injuries, 1990-2015: a systematic analysis for the Global Burden of Disease Study 2015. Lancet 388, 1545-1602. doi: 10.1016/S0140-6736(16)31678-6

Gerber, M., Boettner, A., Seidel, B., Lammert, A., Bar, J., Schuster, E., et al. (2005). Serum resistin levels of obese and lean children and adolescents: biochemical analysis and clinical relevance. J. Clin. Endocrinol. Metab. 90, 4503-4509. doi: 10.1210/jc.2005-0437

German, J. P., Wisse, B. E., Thaler, J. P., Oh, I. S., Sarruf, D. A., Ogimoto, K., et al. (2010). Leptin deficiency causes insulin resistance induced by uncontrolled diabetes. Diabetes 59, 1626-1634. doi: 10.2337/db09-1918

Gharibeh, M. Y., Al Tawallbeh, G. M., Abboud, M. M., Radaideh, A., Alhader, A. A., and Khabour, O. F. (2010). Correlation of plasma resistin with obesity and insulin resistance in type 2 diabetic patients. Diabetes Metab. 36, 443-449. doi: 10.1016/j.diabet.2010.05.003

Gregor, M. F., and Hotamisligil, G. S. (2011). Inflammatory mechanisms in obesity. Annu. Rev. Immunol. 29:415-445. doi: 10.1146/annurev-immunol-031210-101322

Gual, P., Gonzalez, T., Gremeaux, T., Barres, R., Le Marchand-Brustel, Y., and Tanti, J. F. (2003). Hyperosmotic stress inhibits insulin receptor substrate-1 function by distinct mechanisms in 3T3-L1 adipocytes. J. Biol. Chem. 278, 26550-26557. doi: 10.1074/jbc.M212273200

Hara, K., Boutin, P., Mori, Y., Tobe, K., Dina, C., Yasuda, K., et al. (2002). Genetic variation in the gene encoding adiponectin is associated with an increased risk of type 2 diabetes in the Japanese population. Diabetes 51, 536-540. doi: $10.2337 /$ diabetes.51.2.536 studies, extracted the data, and wrote the part of the manuscript. QL and ML extracted the data and wrote the part of the manuscript. JY, DZ, and CZ reviewed and edited the manuscript. JD directed the project, contributed to the discussion, reviewed, and edited the manuscript. JD had full access to all the data in the study and had final responsibility for the decision to submit for publication. KS and YL contributed equally to this work.

\section{FUNDING}

This work was supported by two grants (to JD) from the National Natural Science Foundation of China (No. 31872791) and Natural Science Foundation of Shandong Province of China (No. ZR2019MC046).

Heilbronn, L. K., Rood, J., Janderova, L., Albu, J. B., Kelley, D. E., Ravussin, E., et al. (2004). Relationship between serum resistin concentrations and insulin resistance in nonobese, obese, and obese diabetic subjects. J. Clin. Endocrinol. Metab. 89, 1844-1848. doi: 10.1210/jc.2003-031410

Higgins, J. P., Altman, D. G., Gotzsche, P. C., Juni, P., Moher, D., Oxman, A. D., et al. (2011). The Cochrane Collaboration's tool for assessing risk of bias in randomised trials. BMJ. 343:d5928. doi: 10.1136/bmj.d5928

Hivert, M. F., Manning, A. K., McAteer, J. B., Dupuis, J., Fox, C. S., Cupples, L. A., et al. (2009). Association of variants in RETN with plasma resistin levels and diabetes-related traits in the Framingham Offspring Study. Diabetes 58, 750-756. doi: $10.2337 / \mathrm{db} 08-1339$

Hivert, M. F., Sullivan, L. M., Fox, C. S., Nathan, D. M., D’Agostino, R. B. Sr., Wilson, P. W., et al. (2008). Associations of adiponectin, resistin, and tumor necrosis factor-alpha with insulin resistance. J. Clin. Endocrinol. Metab. 93, 3165-3172. doi: 10.1210/jc.2008-0425

Hotamisligil, G. S., Shargill, N. S., and Spiegelman, B. M. (1993). Adipose expression of tumor necrosis factor-alpha: direct role in obesity-linked insulin resistance. Science 259, 87-91. doi: 10.1126/science.7678183

Jung, H. S., Youn, B. S., Cho, Y. M., Yu, K. Y., Park, H. J., Shin, C. S., et al. (2005). The effects of rosiglitazone and metformin on the plasma concentrations of resistin in patients with type 2 diabetes mellitus. Metab. Clin. Exp. 54, 314-320. doi: 10.1016/j.metabol.2004.05.019

Kaplon-Cieslicka, A., Postula, M., Rosiak, M., Peller, M., Kondracka, A., Serafin, A., et al. (2015). Association of adipokines and inflammatory markers with lipid control in type 2 diabetes. Pol. Arch. Med. Wewn. 125, 414-423. doi: 10.20452/pamw.2880

Kaser, S., Kaser, A., Sandhofer, A., Ebenbichler, C. F., Tilg, H., and Patsch, J. R. (2003). Resistin messenger-RNA expression is increased by proinflammatory cytokines in vitro. Biochem. Biophys. Res. Commun. 309, 286-290. doi: 10.1016/j.bbrc.2003.07.003

Kawamura, R., Doi, Y., Osawa, H., Ninomiya, T., Hata, J., Yonemoto, K., et al. (2010). Circulating resistin is increased with decreasing renal function in a general Japanese population: the Hisayama Study. Nephrol. Dial. Transplant. 25, 3236-3240. doi: 10.1093/ndt/gfq155

Kusminski, C. M., McTernan, P. G., and Kumar, S. (2005). Role of resistin in obesity, insulin resistance and type II diabetes. Clin. Sci. 109, 243-256. doi: 10.1042/CS20050078

Lois, K., and Kumar, S. (2009). Obesity and diabetes. Endocrinol. Nutr. 56, 38-42. doi: 10.1016/S1575-0922(09)73516-8

Luo, Z., Zhang, Y., Li, F., He, J., Ding, H., Yan, L., et al. (2009). Resistin induces insulin resistance by both AMPK-dependent and AMPKindependent mechanisms in HepG2 cells. Endocrine 36, 60-69. doi: 10.1007/s12020-009-9198-7

Mabrouk, R., Ghareeb, H., Shehab, A., Omar, K., El-Kabarity, R. H., Soliman, D. A., et al. (2013). Serum visfatin, resistin and IL-18 in A group of Egyptian obese diabetic and non diabetic individuals. Egypt. J. Immunol. 20, 1-11. 
Maeda, N., Shimomura, I., Kishida, K., Nishizawa, H., Matsuda, M., Nagaretani, H., et al. (2002). Diet-induced insulin resistance in mice lacking adiponectin/ACRP30. Nat. Med. 8, 731-737. doi: 10.1038/nm724

Minn, A. H., Patterson, N. B., Pack, S., Hoffmann, S. C., Gavrilova, O., Vinson, C., et al. (2003). Resistin is expressed in pancreatic islets. Biochem. Biophys. Res. Commun. 310, 641-645. doi: 10.1016/j.bbrc.2003.09.061

Mojiminiyi, O. A., and Abdella, N. A. (2007). Associations of resistin with inflammation and insulin resistance in patients with type 2 diabetes mellitus. Scand. J. Clin. Lab. Invest. 67, 215-225. doi: 10.1080/00365510601032532

Muse, E. D., Obici, S., Bhanot, S., Monia, B. P., McKay, R. A., Rajala, M. W., et al. (2004). Role of resistin in diet-induced hepatic insulin resistance. J. Clin. Invest. 114, 232-239. doi: 10.1172/JCI200421270

Owecki, M., Miczke, A., Nikisch, E., Pupek-Musialik, D., and Sowinski, J. (2011). Serum resistin concentrations are higher in human obesity but independent from insulin resistance. Exp. Clin. Endocrinol. Diabetes 119, 117-121. doi: 10.1055/s-0030-1263111

Park, H., Hasegawa, G., Obayashi, H., Fujinami, A., Ohta, M., Hara, H., et al. (2006). Relationship between insulin resistance and inflammatory markers and anti-inflammatory effect of losartan in patients with type 2 diabetes and hypertension. Clin. Chim. Acta 374, 129-134. doi: 10.1016/j.cca.2006.06.004

Parreno Caparros, E., Illan Gomez, F., Gonzalvez Ortega, M., Orea Soler, I., Perez Paredes, M., Lozano Almela, M. L., et al. (2017). Resistin in morbidly obese patients before and after gastric bypass surgery. Nutr. Hosp. 34, 1333-1337. doi: $10.20960 /$ nh. 1028

Patel, L., Buckels, A. C., Kinghorn, I. J., Murdock, P. R., Holbrook, J. D., Plumpton, C., et al. (2003). Resistin is expressed in human macrophages and directly regulated by PPAR gamma activators. Biochem. Biophys. Res. Commun. 300, 472-476. doi: 10.1016/S0006-291X(02)02841-3

Plomgaard, P., Bouzakri, K., Krogh-Madsen, R., Mittendorfer, B., Zierath, J. R., and Pedersen, B. K. (2005). Tumor necrosis factor-alpha induces skeletal muscle insulin resistance in healthy human subjects via inhibition of Akt substrate 160 phosphorylation. Diabetes 54, 2939-2945. doi: 10.2337/diabetes.54.10.2939

Roden, M., Krssak, M., Stingl, H., Gruber, S., Hofer, A., Furnsinn, C., et al. (1999). Rapid impairment of skeletal muscle glucose transport/phosphorylation by free fatty acids in humans. Diabetes 48, 358-364. doi: 10.2337/diabetes.48.2.358

Roden, M., Price, T. B., Perseghin, G., Petersen, K. F., Rothman, D. L., Cline, G. W., et al. (1996). Mechanism of free fatty acid-induced insulin resistance in humans. J. Clin. Invest. 97, 2859-2865. doi: 10.1172/JCI118742

Sah, S. P., Singh, B., Choudhary, S., and Kumar, A. (2016). Animal models of insulin resistance: a review. Pharmacol. Rep. 68, 1165-1177. doi: 10.1016/j.pharep.2016.07.010

Silswal, N., Singh, A. K., Aruna, B., Mukhopadhyay, S., Ghosh, S., and Ehtesham, N. Z. (2005). Human resistin stimulates the pro-inflammatory cytokines TNFalpha and IL-12 in macrophages by NF-kappaB-dependent pathway. Biochem. Biophys. Res. Commun. 334, 1092-1101. doi: 10.1016/j.bbrc.2005.06.202

Stang, A. (2010). Critical evaluation of the Newcastle-Ottawa scale for the assessment of the quality of nonrandomized studies in meta-analyses. Eur. J. Epidemiol. 25, 603-605. doi: 10.1007/s10654-010-9491-Z
Stepien, M., Rosniak-Bak, K., Paradowski, M., Misztal, M., Kujawski, K., Banach, M., et al. (2011). Waist circumference, ghrelin and selected adipose tissue-derived adipokines as predictors of insulin resistance in obese patients: preliminary results. Med. Sci. Monitor 17, Pr13-Pr18. doi: 10.12659/MSM.882030

Stepien, M., Stepien, A., Wlazel, R. N., Paradowski, M., Rizzo, M., Banach, M., et al. (2014). Predictors of insulin resistance in patients with obesity: a pilot study. Angiology 65, 22-30. doi: 10.1177/0003319712468291

Steppan, C. M., Bailey, S. T., Bhat, S., Brown, E. J., Banerjee, R. R., Wright, C. M., et al. (2001). The hormone resistin links obesity to diabetes. Nature 409, 307-312. doi: 10.1038/35053000

Steppan, C. M., Wang, J., Whiteman, E. L., Birnbaum, M. J., and Lazar, M. A. (2005). Activation of SOCS-3 by resistin. Mol. Cell. Biol. 25, 1569-1575. doi: 10.1128/MCB.25.4.1569-1575.2005

Stern, S. E., Williams, K., Ferrannini, E., DeFronzo, R. A., Bogardus, C., and Stern, M. P. (2005). Identification of individuals with insulin resistance using routine clinical measurements. Diabetes 54, 333-339. doi: 10.2337/diabetes.54.2.333

Tokuyama, Y., Osawa, H., Ishizuka, T., Onuma, H., Matsui, K., Egashira, T., et al. (2007). Serum resistin level is associated with insulin sensitivity in Japanese patients with type 2 diabetes mellitus. Metab. Clin. Exp. 56, 693-698. doi: 10.1016/j.metabol.2006.12.019

Ursini, F., Naty, S., and Grembiale, R. D. (2010). Infliximab and insulin resistance. Autoimmun. Rev. 9, 536-539. doi: 10.1016/j.autrev.2009.12.014

Wang, H., Chu, W. S., Hemphill, C., and Elbein, S. C. (2002). Human resistin gene: molecular scanning and evaluation of association with insulin sensitivity and type 2 diabetes in Caucasians. J. Clin. Endocrinol. Metab. 87, 2520-2524. doi: $10.1210 /$ jcem.87.6.8528

Weigert, C., Hennige, A. M., Lehmann, R., Brodbeck, K., Baumgartner, F., Schauble, M., et al. (2006). Direct cross-talk of interleukin-6 and insulin signal transduction via insulin receptor substrate-1 in skeletal muscle cells. J. Biol. Chem. 281, 7060-7067. doi: 10.1074/jbc.M509782200

Yin, J., Gao, H., Yang, J., Xu, L., and Li, M. (2012). Measurement of salivary resistin level in patients with type 2 diabetes. Int. J. Endocrinol. 2012:359724. doi: 10.1155/2012/359724

Zaidi, S. I., and Shirwany, T. A. (2015). Relationship of serum resistin with insulin resistance and obesity. J. Ayub Med. Coll. 27, 552-555.

Conflict of Interest: The authors declare that the research was conducted in the absence of any commercial or financial relationships that could be construed as a potential conflict of interest.

Copyright (C) 2019 Su, Li, Zhang, Yuan, Zhang, Liu, Song, Lin, Li and Dong. This is an open-access article distributed under the terms of the Creative Commons Attribution License (CC BY). The use, distribution or reproduction in other forums is permitted, provided the original author(s) and the copyright owner(s) are credited and that the original publication in this journal is cited, in accordance with accepted academic practice. No use, distribution or reproduction is permitted which does not comply with these terms. 